\title{
Prospects for Inclusive Jet Cross-Section Measurement with Early Data at ATLAS
}

\author{
Dan Clements * \\ University of Glasgow - Department of Physics and Astronomy \\ Kelvin Building, Glasgow, G12 8QQ - UK
}

\begin{abstract}
The inclusive jet cross-section was studied using the event generator $\mathrm{NLOJET}^{++}$for a number of PDF sets (CTEQ6.1) in order to obtain an understanding of the uncertainties introduced by PDFs and renormalisation/factorisation scales on the theoretical crosssection. The error arising from the jet energy scale calibration was also investigated.
\end{abstract}

\section{Introduction}

The LHC will collide protons at a higher centre of mass energy $(\sqrt{s}=14 T e V)$ and luminosity $\left(L=10^{34} \mathrm{~cm}^{-2} \mathrm{~s}^{-1}\right)$ than previously achieved, providing the opportunity to perform new physics searches (e.g SUSY) and precision tests of the Standard Model.

The inclusive jet cross-section at ATLAS is sensitive to compositeness (quarksubstructure) which could be detected by comparing data with next to leading-order (NLO) QCD predictions. This process however needs to be performed carefully as poorly understood experimental and theoretical errors can lead to false signals of new physics. An understanding of these errors is hence essential in the search for physics beyond the standard model.

The inclusive jet cross-section describes the probability of obtaining a jet with a given transverse momentum. For a given event all jets within acceptance are included in the cross-section. The predicted crosssection for ATLAS using NLOJET ${ }^{++}$[3]

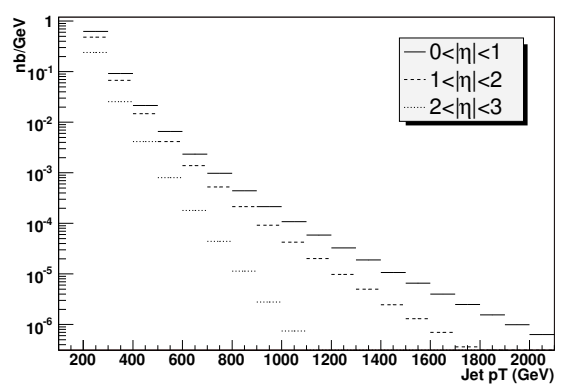

Figure 1: Inclusive jet cross-section for three pseudorapidity ranges at ATLAS $,(\sqrt{s}=14 T e V), \quad \mu_{r / f}=$ $P_{T} / 2,\left(\right.$ NLOJET $^{++}$, CTEQ6.1) and CTEQ6.1 PDF [1] is given in Fig 1.

\section{Theoretical Errors}

\section{$2.1 \quad$ PDFs}

PDFs are parameterised and then fit using global analyses which rely mainly on deep inelastic-scattering (DIS) data. Hence in creating a best fit PDF there is a degree of uncertainty which leads to a systematic error on cross-section predictions. The CTEQ group have attempted to quantify this uncertainty by producing a set of 40 error PDF sets along with a best fit (CTEQ6.1)[1]. The error on an observable dependent on PDFs is found by

*On behalf of the ATLAS collaboration. 
calculating the observable for each PDF set and looking at its fluctuations. For inclusive jet measurements it is found that of the 40 PDF error sets, sets 29 and 30 dominate the error on the observable at high $P_{T}$ and hence only these two sets need to be considered [2]. It is interesting to note that sets 29 and 30 relate to changes in the gluon distribution at high Bjorken-x which is poorly constrained from DIS data owing to the gluon being electrically neutral.

The inclusive jet-cross section was calculated using the central (best-fit) PDF and the error sets 29 and 30 over 3 regions in pseudorapidity $(\eta)$. The proportional error (O(error $\mathrm{PDF})-\mathrm{O}($ central $)) / \mathrm{O}$ (central) was calculated in each case and is shown in Fig 2. For a jet of $P_{T} 1 T e V$ in central regions $0<\eta<1$ the error on the cross-section due to PDFs is $10-15 \%$, the uncertainty in forward regions which probe the high-x region of the PDFs is considerably higher $(\sim 40 \%)$.

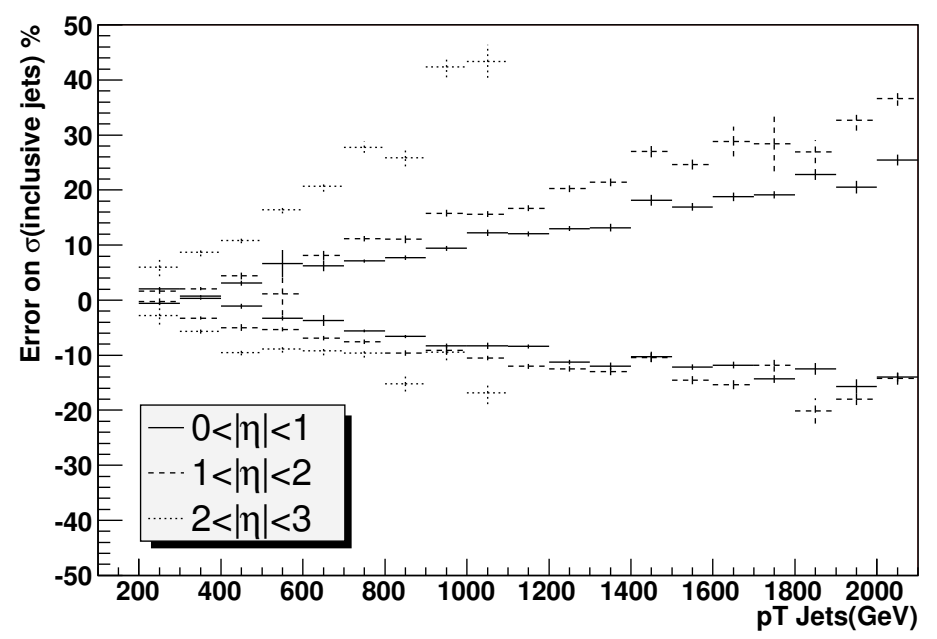

Figure 2: PDF errors on inclusive jet cross-section for three pseudorapidity regions,based on CTEQ6.1 error sets 29,30 in comparison with the best fit set. $\mu_{r / f}=$ $P_{T} / 2,\left(\right.$ NLOJET $^{++}$, CTEQ6.1)

\subsection{Renormalisation And Factorisation Scales}

The renormalisation and factorisation scale $\left(\mu_{r}, \mu_{f}\right)$ dependence of predictions of jet observables comes about owing to the perturbative calculation being carried out to a finite order. As an estimate of the error due to this $\mu_{r}$ and $\mu_{f}$ were varied between $P_{T} / 2 \rightarrow 2 P_{T}$ and the change in the predicted inclusive jet cross section recorded.

The renormalisation and factorisation scale errors were found to be fairly stable with increasing $P_{T}$ of the jet at around $5-10 \%$. This suggests that the renormalisation and factorisation scales will be important at low $P_{T}$ where the PDF errors are small but will not contribute greatly to the overall error at higher $P_{T}$ where PDF errors can become very large. 


\section{$3 \quad$ Experimental Errors}

\subsection{Jet Energy Scale}

The energy of a jet is measured using both electromagnetic and hadronic calorimetry. This method of measurement introduces two distinct types of error to the measured jet energy. The first is a sampling error, which causes a gaussian like smearing of the jet energy around its true value. The second which will be studied here is a systematic calibration error which causes a shift of the measured peak of the jet energy (after smearing) with respect to the true jet energy.

Owing to the strong dependence of the inclusive jet cross-section on the jet energy, jet energy scale uncertainty can create significant errors. This effect was studied by introducing a systematic jet energy scale error to jets after they had been reconstructed within NLOJET $^{++}$and comparing the generated inclusive jet cross-section to an unshifted spectrum. This was carried out for errors on the jet energy scale of $1 \%, 5 \%$ and $10 \%$ the results being shown in Fig 3.

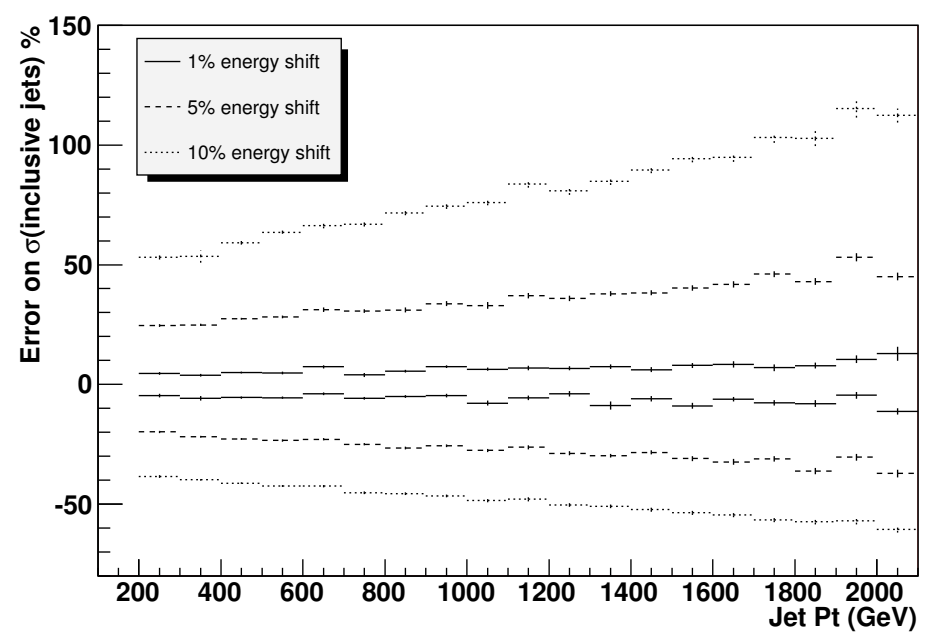

Figure 3: Jet Energy Scale errors of $1 \%, 5 \%$ and $10 \%$ on the inclusive jet cross-section, $\mu_{r / f}=$ $P_{T} / 2,\left(\mathrm{NLOJET}^{++}, \mathrm{CTEQ6} .1\right)$

The error on the the inclusive jet cross-section is seen to grow with increasing jet energy. This is expected as a $1 \%$ error on jet energy constitues a larger absolute error on a high $P_{T}$ jet than on a low $P_{T}$ jet leading to an increased uncertainty in the inclusive jet cross-section. At a jet energy scale uncertainty of $1 \%$, the error on the inclusive jet cross-section is seen to vary from $5 \%$ and $10 \%$ for jet energies between $200 \mathrm{GeV}$ and $2 \mathrm{TeV}$. Similarly a $10 \%$ variation in jet energy scale leads to an error of between $50 \%$ and $120 \%$ over the same range. 


\subsection{Statistical Errors}

The statistical errors on the inclusive jet cross-section at $1 \mathrm{fb}^{-1}$ are of order $1 \%$ in the central region and $5 \%$ in the forward region for a jet of $P_{T} \sim 1 \mathrm{TeV}$. This suggests that at this integrated luminosity the error sources considered so far are statistically significant and will be so with early data.

\section{Improving Gluon PDFs with ATLAS data}

ATLAS jet data could be used in a global PDF fit in order to help constrain the high-x gluon. The recent development of integration grid-techniques such as NLOGRID [4] and FASTNLO [5] allow for a NLO cross-section to be rapidly calculated for a varying PDF. This hence allows jet data to be introduced to existing PDF global fits used by MRST and CTEQ.

A study based on pseudo-data suggests that ATLAS jet data will be able to constrain the high-x gluon if it can achieve a jet energy scale uncertainty of $\sim 1 \%$. At JES uncertainties of $\sim 3 \%$ the benefits of including ATLAS data into global PDF fits becomes negligible.

\section{Summary}

Jet data at ATLAS will provide tests of QCD and the opportunity to look for evidence of new physics. However a good control of both theoretical and experimental errors is vital in order to have confidence in any results. The theoretical uncertainty (for high pT jets) is dominated by uncertainty in the high-x gluon PDF, whilst the experimental errors are dominated by uncertainty in the jet energy scale. With early data the JES is likely to be poorly constrained $(5-10 \%$ for jets below $1 \mathrm{TeV})$, which will seriously limit the physics potential of the data. The use of in situ-calibration techniques and a growing understanding of the detector should however lead eventually to a better constrained JES 1-2\% (below $1 \mathrm{TeV}$ ) with the potential to constrain the high-x gluon.

\section{Acknowledgments}

The author would like to thank: C.Buttar, C.Gwenlan, T.Carli, A.Cooper-Sarkar and P.Francavilla.

\section{Bibliography}

\section{References}

[1] J Pumplin et al., arXiv: hep-ph 0201195, (2004).

[2] D Stump et al,, arXiv: hep-ph 0303013, (2003).

[3] Z Nagy, Phys Rev Lett 88, 122003, (2002).

[4] T Carli et al,, arXiv: hep-ph 0510324 (2005).

[5] T Kluge et al,, arXiv: hep-ph 0609285 (2006).

[6] Slides:

http: //indico . cern. $\mathrm{ch} /$ contributionDisplay . py? contribId=228\&sessionId=6\&conf Id=9499 